\title{
Yacon: benefícios à saúde e aplicações tecnológicas
}

\author{
Yacon: health benefits and technological applications
}

\author{
Ana Paula Gusso ${ }^{I^{*}}$ Paula Mattanna $^{\mathrm{I}}$ Neila Richards ${ }^{\mathrm{II}}$
}

\section{- REVISÃO BIBLIOGRÁFICA -}

\section{RESUMO}

No início dos anos 90, foi introduzido na cultura Brasileira o plantio da batata yacon, uma raiz tuberosa, que vem despertando grande interesse por parte dos pesquisadores, devido as suas propriedades nutricionais e tecnológicas. Essa raiz caracteriza-se principalmente por ser rica em frutanos $e$ ácidos fenólicos, apresentando efeito prebiótico e antioxidante, respectivamente. Muitos estudos in vivo e in vitro já foram realizados, utilizando este tubérculo e seus derivados, comprovando suas atividades benéficas à saúde, além deter grande potencial na tecnologia do desenvolvimento de novos produtos alimentícios. A presente revisão objetivou agrupar alguns desses estudos, tanto na área da saúde como na da tecnologia de alimentos. $O$ potencial prebiótico, redução do índice glicêmico e a melhora na saúde óssea são os efeitos que se destacam nas pesquisas in vivo. Percebe-se também uma tendência na elaboração de produtos de panificação utilizando-se a farinha de yacon, e os resultados apresentam-se satisfatórios, tanto nas propriedades tecnológicas quanto nas avaliações sensoriais.

Palavras-chave: alimento funcional, Smallanthus sonchifolius, saúde e tecnologia de alimentos.

\section{ABSTRACT}

In the early nineties was introduced in Brazil the yacon potato planting. This tuberous roots have been attracting interest from researchers due to their nutritional and technological properties.Yacon is its mainly characterized by being rich in fructans and phenolic acids, with prebiotic and antioxidant effects, respectively. Many studies in vivo and in vitro were carried out with yacon and products produced from it, showing its health benefits, besides having great technological potential for developing new food products. This review aimed to outline some of these studies, in both health and food technology. The prebiotic potential, glycemic index reduction and improvement of bone health are the effects that stand out in the in vivo researches. It can be noted a trend for bakery products formulations using yacon flour, and the outcomes were positive for both technological properties as well as for sensory evaluations.

Key words: functional food, Smallanthus sonchifolius, health and food technology.

\section{INTRODUÇÃO}

O interesse mundial na produção da batata yacon, principalmente pelas indústrias alimentícias e farmacêuticas, deve-se ao fato de essa raiz tuberosa ser considerada um alimento funcional, apresentando em sua composição compostos bioativos que oferecem benefícios à saúde (VANINI et al., 2009). Dentre os componentes bioativos presentes na yacon e seus derivados, destacam-se os frutanos, do tipo inulina, e fruto-oligossacarídeos (FOS) (SANTANA \& CARDOSO, 2008). Também se fazem presentes compostos fenólicos, tais como ácido clorogênico, ácido ferúlico e ácido cafeico (TAKENAKA et al., 2003), além de flavonoides, como a quercetina (VALENTOVÁ \& ULRICHOVÁ, 2003).

Os derivados desse tubérculo também apresentam grandes potencialidades na medicina preventiva. OLIVEIRA et al. (2013) demonstraram que o consumo de extrato aquoso de raízes de yacon pode aumentar o colesterol bom (HDL, High-density lipoprotein), reduzir o colesterol total, triglicerídeos e outras lipoproteínas. Estes resultados foram obtidos através de análises sorológicas realizadas em ratos

'Programa de Pós-graduação em Ciência e Tecnologia dos Alimentos, Universidade Federal de Santa Maria (UFSM), Av. Roraima, 1000,

Camobi, 97105-900, Santa Maria, RS, Brasil. E-mail: anapgusso@gmail.com. *Autor para correspondência.

IIDepartamento de Tecnologia e Ciência dos Alimentos, UFSM, Santa Maria, RS, Brasil. 
machos adultos (Wistar), alimentados com o extrato. $\mathrm{Na}$ pesquisa conduzida por TOSTES et al. (2014), os autores constataram que o consumo de alimentos fortificados com farinha de yacon melhorou o sistema imune de crianças com idade entre dois e cinco anos.

As farinhas e extratos da yacon vêm sendo utilizadas na tecnologia de alimentos como fontes de fibras e têm demostrado resultados relativamente satisfatórios nas análises físico-químicas e sensoriais. Dentre os produtos fabricados com os derivados da yacon, estão o apresuntado (TEIXEIRA, 2011), iogurte light (VASCONCELOS, 2010), doce adicionado de diferentes geleificantes (MALDONADO \& SINGH, 2008), bolo adicionado de farinha de yacon (ROSA et al., 2009) e pães de forma (ROLIM et al., 2010).

Diante das recentes pesquisas envolvendo a batata yacon e seus derivados, o objetivo deste trabalho foi realizar uma revisão sistemática da literatura para demonstrar as potencialidades da yacon, tanto na prevenção de doenças quanto na aplicação da tecnologia de alimentos.

Características da Yacon

A yacon (Smallanthus sonchifolius, anteriormente denominada na literatura científica de Polymnia sonchifolius) é uma planta da espécie Asteraceae, originária da região andina e introduzida no Brasil por descendentes japoneses por volta de 1989. Em meados dos anos 2000, teve início o consumo expressivo dessa raiz, tornando-se conhecida popularmente como batata yacon ou batata diet (OLIVEIRA \& NISHIMOTO, 2005; SANTANA \& CARDOSO, 2008).

Segundo SEMINARIO \& VALDERRAMA (2003), a yacon é uma planta perene e herbácea que mede entre um e 2,5 metros de altura e apresenta um sistema de raiz composto de 4 a 20 tubérculos. O peso das raízes de reserva pode variar de 50 a 1000 gramas, porém, mais comumente varia entre 300 e 600 gramas. Uma planta produz em média entre 2 e $4 \mathrm{~kg}$ de raízes de reserva. A colheita das raízes tuberosas para consumo é realizada por volta de 10 e 12 meses após o plantio, quando a parte aérea está totalmente seca (OLIVEIRA \& NISHIMOTO, 2004).

Poucas espécies se adaptam a diferentes ecossistemas como a yacon. Segundo ROBINSON (1997), essa planta pode ser cultivada em altitudes variadas, como ocorre na Bolívia, Equador e Peru, onde é cultivada em altitudes entre $900 \mathrm{~m}$ e $3.500 \mathrm{~m}$ acima do nível do mar; na Argentina, é cultivada entre $600 \mathrm{~m}$ e $2.500 \mathrm{~m}$; no Brasil, entre $600 \mathrm{~m}$ e $800 \mathrm{~m}$; ou ao nível do mar, como no Japão e na Nova Zelândia.

O consumo da yacon varia, mas ela é consumida preferencialmente in natura. Tem sabor adocicado e refrescante. Podem ser cozidas ou desidratadas na forma de chips. Quando o suco da yacon é colocado para ferver, transforma-se em blocos de açúcar e é chamado de "chancaca" pelos nativos dos Andes (SEMINARIO \& VALDERRAMA, 2003).

Yacon fonte de frutanos e compostos fenólicos

Diferente da maioria das raízes que armazenam carboidratos na forma de amido, a yacon e várias plantas da família Asteraceae armazenam os carboidratos na forma de frutano (GENTA et al., 2009). Os frutanos são carboidratos de reserva na forma de polímeros de D-frutose, unidos por ligações tipo $\beta(2 \rightarrow 1)$, e apresentam uma glicose na extremidade da cadeia (ROBERFROID, 2007). Dependendo do comprimento da cadeia, definido pelo número de unidades de monossacarídeos, denominado grau de polimerização (DP), temse a inulina e os fruto-oligossacarídeos (SAAD, 2006). O grau de polimerização da inulina varia entre 2 e 60 e dos fruto-oligossacarídeos entre 2 e 9 (BIEDRZYCKA \& BIELECKA, 2004).

Os frutanos são capazes de resistir à hidrólise pelas enzimas digestivas do corpo humano e, dessa forma, passam por meio do trato digestivo sem serem metabolizados, fornecendo baixo conteúdo energético $\left(1,5 \mathrm{kcal} / \mathrm{g}^{-1}\right)$ e exercendo funções semelhantes à fibra alimentar (AYBAR et al., 2001; GENTA et al., 2009).

De acordo com VILHENA et al. (2000), os órgãos subterrâneos da yacon, recém colhida, contêm de $60 \%$ a $70 \%$ de frutanos do tipo inulina e fruto-oligossacarídeos, respectivamente. A maior concentração de FOS na yacon é observada quando a raiz é colhida no período entre a $31^{\mathrm{a}}$ e $35^{\mathrm{a}}$ semanas após o plantio (OLIVEIRA \& NISHIMOTO, 2004). Ao atingir a maturidade, a tendência dos FOS é se degradarem em compostos menores como frutose, glicose e sacarose (GRAEF et al., 2004).

O fenômeno de despolimerização, ou seja, conversão de FOS em monômeros, foi verificado durante o período de armazenamento das raízes de yacon, nos estudos realizados por ASAMI et al. (1991), SEMINARIO \& VALDERRAMA (2003) e GRAEF et al. (2004). Este processo de mudança na composição química ocorre devido à ação da enzima frutano hidrolase $(\mathrm{FH})$, que converte os FOS em glicose, frutose e sacarose. No entanto, a velocidade desta conversão é mais lenta se a yacon é armazenada em temperaturas de refrigeração. Em todos os trabalhos, verificou-se que as menores temperaturas permitiram uma melhor conservação dos FOS. Portanto, quando usadas como fonte de FOS, as raízes de yacon devem ser consumidas logo após a 
colheita ou armazenadas sob refrigeração, impedindo assim a conversão dos FOS (GRAEF et al., 2004; MANRIQUE et al., 2005).

Pesquisadores também confirmaram a presença de compostos fenólicos tanto nas folhas quanto nas raízes tuberosas de yacon. Alguns dos compostos identificados são o ácido cafeico e seus derivados, ácido clorogênico e L-triptofano. Em comparação a outras raízes e tubérculos, as raízes da yacon possuem elevada quantidade de compostos fenólicos, cerca de $200 \mathrm{mg} / 100 \mathrm{~g}^{-1}$ de matéria fresca comestível (SIMONOVSKA et al. 2003; VALENTOVÁ \& ULRICHOVÁ 2003; TAKENAKA, 2003).

\section{Aspectos funcionais da batata e seus derivados}

$O$ efeito benéfico de determinados tipos de alimentos sobre a saúde do hospedeiro é conhecido há muito tempo (OLIVEIRA, 2009). Apesar disso, o termo alimento funcional foi definido inicialmente no Japão, durante a década de 1980, como "alimento para uso específico de saúde”. O conceito contemporâneo refere-se aos alimentos ou nutrientes cuja ingestão leva a importantes mudanças fisiológicas ao organismo, isoladas ou distintas daquelas associadas ao seu papel como nutriente (FOOD AND DRUG ADMINISTRATION, 2004). Com base em sua composição, surge a hipótese de que a yacon pode ser considerada um alimento funcional pelo seu conteúdo de substâncias bioativas como os FOS, inulina e compostos fenólicos (RIBEIRO, 2008).

Quando ingeridos pelo homem, os frutanos atingemo cólon, onde são fermentados pela microbiota, composta principalmente de bifidobactérias. Pela ação dessas bactérias, são liberados ácidos graxos de cadeia curta (short chain fatty acids, SCFA) como ácido acético, propiônico e butírico, além de $\mathrm{CO}_{2}, \mathrm{CH}_{4}$ e $\mathrm{H}_{2}$ (WANG \& GIBSON, 1993). Com a produção de ácidos de cadeia curta, a absorção de cálcio, ferro e magnésio é facilitada. Com $15 \mathrm{~g}$ de FOS por dia, a absorção de $\mathrm{Ca}^{++}$é aumentada em 10,8\% em humanos (GIBSON \& ROBERFROID, 1995).

No Brasil, de todos os benefícios atribuídos à yacon, a maior importância refere-se à redução da glicemia e ao auxílio na redução da obesidade (SILVA \& CANDIDO, 2004). Sabe-se que a maior parte dos açúcares solúveis na yacon são frutoses, e este monossacarídeo não é dependente de insulina para ser captado pelas células para sua utilização, não elevando dessa forma os níveis de glicose no sangue. (SEMINÁRIO\&VALDERRAMA, 2003; RIBEIRO, 2008).

Além disso, estudos sugerem que os FOS e a inulina presentes na yacon, podem atuar na inibição dos estágios iniciais do câncer de cólon, reduzir os níveis de colesterol, pressão arterial e glicose no sangue. Também atuam melhorando a regularização da função intestinal, através da eliminação de bactérias patogênicas e putrefativas por efeito da multiplicação das bifidobactérias (GIBSON \& ROBERFROID, 1995; AYBAR et al., 2001;GUIGOZ et al., 2002; PEREIRA \& GIBSON, 2002; COUNDRAY et al., 2003; ALBUQUERQUE \& ROLIM, 2011; LOBO et al., 2011; DE MOURA et al., 2012).

$\mathrm{O}$ efeito antioxidante das raízes de yacon foi avaliado no estudo realizado por CASTRO et al. (2012). Os autores relataram que a atividade antioxidante permanece nas amostras secas e que a retenção da atividade antioxidante é dependente do método de secagem utilizado e da geometria das amostras. Neste estudo, confirmou-se que temperaturas de secagem próximas a $50^{\circ} \mathrm{C}$ e batatas cortadas na forma de tiras, resultam em uma melhor retenção da atividade antioxidante da yacon. De acordo com MAYDATA (2002), os compostos fenólicos têm como propriedades gerais serem antioxidantes. Dessa forma, exercem efeitos quelantes e modulam as atividades de vários sistemas enzimáticos, de modo a atuar majoritariamente na dieta, como elementos que promovem saúde ante aos fatores químicos e físicos estressantes para o organismo.

Diversas pesquisas foram e estão sendo realizadas com o intuito de avaliar as propriedades funcionais da yacon e seus derivados. Algumas delas podem ser visualizadas na tabela 1 .

Potencialidades da yacon na tecnologia de alimentos

O modo de consumo da yacon pode variar de acordo com sua utilização, mas é consumida preferencialmente in natura, por apresentar sabor adocicado e refrescante (SEMINARIO \& VALDERRAMA, 2003). Nos mercados andinos, a yacon é classificada como fruta e é exposta juntamente com as maçãs, os abacates e os abacaxis, em vez de ser colocada com as batatas e outras culturas de tubérculos e raízes (VALENTOVÁ \& ULRICHOVÁ, 2003).

A yacon tem a aparência de batata doce e o seu sabor tem sido descrito como levemente adocicado, lembrando o sabor da melancia, tendo crocância de uma maçã recém-colhida. A casca da yacon apresenta uma cor que varia de marrom a uma tonalidade arroxeada, enquanto a porção comestível pode ser branca, amarela, laranja ou roxa, dependendo da quantidade de pigmentos presentes na raiz (MANRIQUE et al., 2005). A desidratação da yacon, para obtenção de farinha tem se mostrado interessante, não só por aumentar a sua vida útil, mas também por facilitar sua incorporação na formulação de bolos, biscoitos, doces, sucos, dentre outros (SILVA, 2007). 
Tabela 1 - Estudos com Yacon e seus derivados sobre os benefícios constatados à saúde.

\begin{tabular}{|c|c|c|c|}
\hline Tipo de estudo & Produto usado & Efeito observado & Referências \\
\hline $\begin{array}{l}\text { Modelo animal (ratos } \\
\text { Wistar machos) com } \\
\text { deficiência de Fe }\end{array}$ & Farinha de yacon & $\begin{array}{l}\text { Melhor biodisponibilidade de } \mathrm{Fe} \text {, redução do } \mathrm{pH} \\
\text { cecal e aumento da produção de ácidos graxos } \\
\text { de cadeia curta, quando comparado a uma fonte } \\
\text { de frutanos purificada. }\end{array}$ & LOBO et al., 2011. \\
\hline in vitro & $\begin{array}{l}\text { Pães com farinha de } \\
\text { yacon }\end{array}$ & $\begin{array}{l}\text { Os pães apresentaram índice glicêmico baixo e } \\
\text { moderado, de acordo com o conteúdo de farinha } \\
\text { adicionado. O potencial prebiótico também foi } \\
\text { observado, devido ao crescimento de bactérias } \\
\text { probióticas durante a fermentação in vitro. }\end{array}$ & ROLIM et al., 2011. \\
\hline $\begin{array}{l}\text { Modelo animal (ratos } \\
\text { Wistar machos) }\end{array}$ & Farinha de yacon & $\begin{array}{l}\text { Melhora da saúde óssea, por aumentar a } \\
\text { resistência à fratura e a retenção de minerais } \\
\text { (cálcio, fósforo e magnésio) nos ossos. }\end{array}$ & RODRIGUES, 2011. \\
\hline $\begin{array}{l}\text { Modelo animal (ratos } \\
\text { Caviaporcellus machos) }\end{array}$ & Farinha de yacon & $\begin{array}{l}\text { Identificação e quantificação de compostos } \\
\text { fenólicos em diferentes variedades de yacon. O } \\
\text { potencial prebiótico foi verificado in vivo, e os } \\
\text { modelos apresentaram vários indicativos benéficos } \\
\text { à saúde intestinal. }\end{array}$ & CAMPOS et al., 2012. \\
\hline $\begin{array}{l}\text { Modelo animal (Ratos } \\
\text { Wistar machos com quatro } \\
\text { semanas de idade) }\end{array}$ & $\begin{array}{l}\text { Extrato seco da raiz de } \\
\text { yacon e uma formulação } \\
\text { simbiótica ( } 1,0 \% \text { de raiz } \\
\text { de yacon e } \\
\text { Lactobacillus casei) }\end{array}$ & $\begin{array}{l}\text { Uma redução significativa na incidência de tumores } \\
\text { no cólon induzidos quimicamente. A ingestão de raiz } \\
\text { yacon pode ter potencial quimiopreventivo contra a } \\
\text { carcinogênese do cólon. }\end{array}$ & MOURA et al., 2012. \\
\hline $\begin{array}{l}\text { Modelo animal } \\
\text { (Camundongos fêmeas) }\end{array}$ & $\begin{array}{l}\text { Raízes de yacon moídas } \\
\text { e liofilizadas }\end{array}$ & $\begin{array}{l}\text { Os resultados apoiam a hipótese de que oconsumo } \\
\text { regular de yacon não exerce efeitos negativos sobre } \\
\text { o sistema imunológico. Ajuda a preservar um } \\
\text { estado anti-inflamatório nas células fagocíticas e } \\
\text { melhora a imunidade da mucosa, possivelmente } \\
\text { evitando os riscos associados com doenças } \\
\text { autoimunes e metabólicas. }\end{array}$ & DELGADO et al., 2012. \\
\hline $\begin{array}{l}\text { Estudo com } 62 \text { idosos com } \\
\text { idade média de } 67 \text { anos }\end{array}$ & Yacon liofilizado (YL) & $\begin{array}{l}\text { A ingestão diária de YL com } 7,4 \mathrm{~g} \text { de FOS por } \\
\text { nove semanas diminuiu significativamente } \\
(4,6 \%) \text { a glicemia sanguínea, mas não reduziu a } \\
\text { concentração de lipídios séricos em idosos. A } \\
\text { resposta imunológica também foi benéfica. }\end{array}$ & SCHEID, 2013. \\
\hline $\begin{array}{l}\text { Modelo animal (ratos } \\
\text { Wistar machos) }\end{array}$ & $\begin{array}{l}\text { Extrato aquoso de raízes } \\
\text { de yacon }\end{array}$ & $\begin{array}{l}\text { O extrato aquoso mostrou-se } \\
\text { eficaz em reverter alterações patológicas, } \\
\text { especialmente a dislipidemia, hiperglicemia e } \\
\text { diabetes mellitus induzida, além de apresentar } \\
\text { função protetora do figado. }\end{array}$ & OLIVEIRA, et al., 2013. \\
\hline $\begin{array}{l}\text { Estudo com } 117 \text { crianças } \\
\text { entre dois e cinco anos de } \\
\text { idade }\end{array}$ & $\begin{array}{l}\text { Farinha de yacon } \\
\text { utilizada na preparação } \\
\text { de doces, bolos e } \\
\text { biscoitos }\end{array}$ & $\begin{array}{l}\text { Os alimentos suplementos com yacon não } \\
\text { melhoraram o estado nutricional de ferro e zinco } \\
\text { nas crianças, contudo, promoveram melhoras } \\
\text { nos efeitos imunológicos, com a maior produção } \\
\text { de IgA secretora de IL-4. }\end{array}$ & TOSTES et al., 2014. \\
\hline
\end{tabular}

Outra opção de comercialização e agregação de valores comerciais à yacon é a produção de sucos, que podem ser facilmente incorporados à dieta de diabéticos e da população em geral. No início da década de 90, foram desenvolvidas no
Japão bebidas a partir da yacon branqueada, moída e submetida à ação de celulases a $45^{\circ} \mathrm{C}$ por duas horas. O suco é utilizado como adoçante em formulações prontas para beber (QUINTEROS, 2000). HISAE et al. (1996) patentearam uma metodologia para preparar 
bebida nutritiva a partir de leite desnatado e suco de yacon fermentado pela ação das bactérias lácticas (Lactocacillus plantarum). Inúmeras pesquisas foram e estão sendo desenvolvidas na área da tecnologia de alimentos utilizando a yacon e seus derivados, algumas delas são exemplificados na tabela 2 .

Tabela 2 - Estudos sobre a utilização da yacon e seus derivados na tecnologia de alimentos.

\begin{tabular}{l} 
Tipo de estudo \\
\hline Aplicação de FY \\
em produtos à
\end{tabular}

em produtos à

base de cereais
Desenvolvimento

de doce

adicionado de

diferentes

geleificantes

Elaboração de

bolo com farinha

de yacon

Desenvolvimento

de massa

alimentícia

funcional

Elaboração de pão de forma

Formulação de bolo de chocolate

Caracterização físico-química e sensorial de iogurte "light"

Elaboração de apresuntado adicionados de farinha e extrato de yacon
Farinha de yacon (FY)

Farinha de yacon e extrato em pó de yacon

Farinha de yacon

Raízes de yacon

Farinha de yacon (FY)

Farinha (FY) e extrato de yacon (EY)
Farinha de yacon funcional em produtos panificados e extrudados mostrou-se viável. Os FOS foram conservados no processamento da Yacon até a obtenção da farinha e permaneceram em concentrações satisfatórias nos produtos, de modo a suprir a ingestão nutricional de acordo com padrões difundidos na literatura.

A formulação de doce com adição de ágarágar $(0,48 \%)$ apresentou-se dentro parâmetros de textura buscados. A análise sensorial mostrou que os doces apresentam resultados similares à amostra de referência.

O bolo formulado com $20 \%$ de FY apresentou-se mais firme e com maior resistência ao corte. A avaliação sensorial do atributo aparência não evidenciou diferença estatística significativa entre os três bolos (padrão, com 10 e 20\% de FY).

As massas alimentícias elaboradas apresentaram boas características (baixo tempo de cozimento, boa textura, aumento de massa satisfatório), mostrando-se semelhantes às massas comerciais de farinha de trigo. Elas tiveram boa aceitação sensorial.

Não houve diferença entre os pães padrão e testes quanto aos atributos porosidade, textura, sabor e qualidade global. Entretanto, o pão com $6 \%$ de farinha de yacon apresentou maior média para $o$ atributo aderência, fato indesejável para a qualidade sensorial de pães.

A adição de 20 e $40 \%$ de FY nos bolos de chocolate influenciou no perfil sensorial e na caracterização cromática, deixando-os mais escuros. O bolo com maior teor de farinha de yacon obteve as maiores notas para os atributos: aroma, gosto doce, maciez, sabor de chocolate e qualidade global.

O uso da FY permitiu a elaboração de um produto com reduzido teor de gordura, baixa caloria e fonte de fibras. Contudo o teste de aceitação sensorial apresentou médias baixas.

A adição de FY e EY em substituição à carne e água, respectivamente, não interferiram na qualidade do produto. De maneira geral, os produtos com concentrações médias de FY e $\mathrm{EY}$, foram mais bem aceitos, inclusive em relação à intenção de compra.
MARANGONI, 2007.

MALDONADO \&

SINGH, 2008.

ROSA et al., 2009.

GONÇALVES, 2010 .

ROLIM et al., 2010.

PADILHA et al., 2010.

VASCONCELOS, 2010.

TEIXEIRA, 2011.

Ciência Rural, v.45, n.5, mai, 2015. 
Segundo SILVA (2007), a indústria de panificação tem investido na tecnologia de alimentos ao elaborar produtos a partir de matérias-primas não convencionais, como a yacon, tendo um perfil nutricional em média de $65 \%$ de carboidrato, $8 \mathrm{~g}$ de proteínas, pobre em lipídeos $(0,52 \mathrm{~g})$ e rico teor de fibra alimentar (3,6g). Considerando tais características, esses produtos apresentam-se como alternativas alimentares de excelente valor nutritivo e funcional.

Os frutanos presentes na yacon não necessitam de insulina para seu metabolismo, um dos fatores que justifica o desenvolvimento de novos produtos utilizando a yacon como alternativa de substituição ao açúcar, destinados a pacientes diabéticos. Esses compostos bioativos, inulina e FOS, são promissores na aplicação clínica, pois, além de melhorar a saúde intestinal, somam benefícios funcionais em indivíduos portadores de diabetes (ALBUQUERQUE \& ROLIM, 2011).

\section{CONCLUSÃO}

As pesquisas relacionadas à área da saúde utilizando a yacon e seus derivados se destacam pelo potencial prebiótico, atividade antioxidante, melhora do sistema imune e redução da glicemia. Em função dos benefícios nutricionais e funcionais que a batata yacon apresenta, a farinha dessa raiz vem sendo desenvolvida e utilizada como ingrediente em alimentos. $\mathrm{O}$ uso da yacon e seus derivados no desenvolvimento de novos produtos alimentícios são promissores, visto que suas propriedades funcionais estão sendo cada vez mais pesquisadas e comprovadas cientificamente.

Percebe-se que há uma tendência na elaboração de produtos de panificação utilizandose a farinha de yacon, e os resultados apresentamse satisfatórios, tanto na tecnologia quanto nas avaliações sensoriais. $\mathrm{O}$ sabor adocicado da yacon, característico dos frutanos, favorece $\mathrm{o}$ desenvolvimento de produtos nesse segmento.

\section{AGRADECIMENTOS}

À Fundação de Amparo à Pesquisa do Estado do Rio Grande do Sul (FAPERGS) pela bolsa de doutorado concedida. Ao Programa de Pós-graduação em Ciência e Tecnologia dos Alimentos, da Universidade Federal de Santa Maria, pelo apoio neste estudo.

\section{REFERÊNCIAS}

ALBUQUERQUE, E.N.; ROLIM, P.M. Potencialidades do yacon (Smallanthus sonchifolius) no diabetes Mellitus. Revista Ciências Médicas, v.20, n.3-4, p.99-108, 2011. Disponível em: $<$ http://200.18.252.94/seer/index.php/cienciasmedicas/article/ viewFile/584/564>. Acesso em: 12 dez. 2013.
ASAMI, T. et al. Fluctuations of oligofructan contents in tubers of yacon (Polymnia sonchifolia) during growth and storage. Japan Journal Soil Science Plant Nutrition, v.62, p.621-627, 1991.

AYBAR, M.J. et al. Hypoglicemic effect if the water extract of Smallantus sonchifolius (yacon) leaves in normal and diabetic rats. Journal of Ethnopharmacol, v.74, n.2, p.33-37, 2001. Disponível em: <http://dx.doi.org/10.1016/S0378-8741(00)00351-2>. Acesso em: 13 nov. 2013. doi: 10.1016/S0378-8741(00)00351-2.

BIEDRZYCKA, E.; BIELECKA, M. Prebiotic effectiveness of fructans of different degrees of polymerization. Trends in Food Science \& Technology, v.15, p.170-175, 2004. Disponível em: $<$ http://dx.doi.org/10.1016/j.tifs.2003.09.014>. Acesso em: 21 de nov. 2013. doi:10.1016/j.tifs.2003.09.014.

CAMPOS, D. et al. Prebiotic effects of yacon (Smallanthus sonchifolius) a source of fructo-oligosaccharides and phenolic compounds with antioxidant activity. Food Chemistry, v.135, p.1592-1599, 2012. Disponível em: <http://dx.doi.org/10.1016/j. foodchem.2012.05.088>. Acesso em: $12 \mathrm{dez} .2013$. doi: 10.1016/j. foodchem.2012.05.088

CASTRO, A. et al. Antioxidants in yacon products and effect of long term storage. Ciência e Tecnologia de Alimentos, v.32, n.3, p.432-435, 2012. Disponível em: <http://dx.doi.org/10.1590/ S0101-20612012005000064>. Acesso em: 15 dez. 2013. doi:10.1590/S0101-20612012005000064

COUNDRAY, C. et al. Effect of soluble or partly soluble dietary fibres supplementation on absorption and balance of calcium, magnesium, iron and zinc in healthy young men. European Journal of Clinical Nutrition, v.51, p.375-380, 2003. Disponível em: <http://europepmc.org/abstract/MED/9192195>. Acesso em: 12 nov. 2013. doi:10.1038/sj.ejen. 1600417.

DE MOURA, N.A. et al. Protective effects of yacon (Smallanthus sonchifolius) intake on experimental colon carcinogenesis. Food and Chemical Toxicology, v.20, p.2902-2910, 2012. Disponível em: <http://dx.doi.org/10.1016/j.fct.2012.05.006>. Acesso em: 24 nov.2013. doi: 10.1016/j.fct.2012.05.006.

DELGADO, G.T.C. et al. Yacon (Smallanthus sonchifolius) derived fructooligo saccharides improves the immune parameters in the mouse. Nutrition Research, v.32, p.884-892, 2012. Disponível em: <http://dx.doi.org/10.1016/j.nutres.2012.09.012>. Acesso em: 23 nov. 2013. doi: 10.1016/j.nutres.2012.09.012.

FOOD AND DRUG ADMINISTRATION. Probiotics. New York, 2004. Disponível em: <http://www.webdietitians.org/Public/ GovernmentAffairs/92_adap1099.cfm>. Acesso em: 06 ago. 2014.

GENTA, S. etal. Yacon syrup: beneficial effects on obesity and insulin resistance in humans. Clinical Nutrition, v.28, p.182-187, 2009. Disponível em: <http://dx.doi.org/10.1016/j.clnu.2009.01.013>. Acesso em: 12 nov. 2013. doi: 10.1016/j.clnu.2009.01.013.

GIBSON, G.R.; ROBERFROID, M.B. Dietary modulation of the human colonic microbiota: introducing the concept of prebiotics. Journal of Nutrition, v.125, n.6, p.1401-1412, 1995. Disponível em: $<$ http://ilri.org/biometrics/Publication/Abstract/Case $\% 20$ study $\% 20$ 17\%20-1.pdf>. Acesso em: 24 nov. 2013.

GONÇALVES, P.V.M. Desenvolvimento de massa alimenticia funcional a base de extrato em pó e farinha de Yacon (Polymnia sonchifolia) e farinha de arroz por processo de extrusão

Ciência Rural, v.45, n.5, mai, 2015. 
termoplástica. 2010. 120f. Dissertação (Mestrado em Tecnologia de Alimentos) - Universidade Estadual de Campinas, Faculdade de Engenharia de Alimentos, Campinas, SP.

GRAEF, S.et al. Effects of post-harvest treatments on the carbohydrate composition of yacon roots in the Peruvian Andes. Field Crops Research, v.86, p.157-65, 2004. Disponível em: $<$ http://dx.doi.org/10.1016/j.fcr.2003.08.003>. Acesso em: 12 nov. 2013. doi: 10.1016/j.fcr.2003.08.003.

GUIGOZ, Y.et al. Effects of oligosaccharides on the fecal flora and non-specific immune system in elderly people. Nutrition of Research, v.22, p.13-25, 2002. Disponível em: <http://dx.doi. org/10.1016/S0271-5317(01)00354-2>. Acesso em: 12 dez. 2013. doi: $10.1016 / \mathrm{S} 0271-5317(01) 00354-2$.

HISAE, T. et al. Preparation of fermented yacon drink. (CL. A23L2/52). JP 08294379A. 1p. 27 apr. 1995, 12 nov. 1996.

LOBO, A.R. et al. Iron bioavailability from ferric pyrophosphate in rats fed with fructan containing yacon (Smallanthus sonchifolius) flour. Food Chemistry, v.126, p.885-891, 2011. Disponível em: $<$ http://dx.doi.org/10.1016/j.foodchem.2010.11.067>. Acesso em: 13 dez. 2013. doi. 10.1016/j.foodchem.2010.11.067.

MALDONADO, S.; SINGH, J.D.C. Efecto de gelificantes en la formulación de dulce de yacón. Ciência e Tecnologia de Alimentos, v.28, n.2, p.429-434, 2008. Disponível em: <http:// dx.doi.org/10.1590/S0101-20612008000200025>. Acesso em: 24 nov. 2013. doi: 10.1590/S0101-20612008000200025.

MANRIQUE, I. et al. Yacon syrup: principles and processing, Series: Conservación y uso de labiodiversidad de raíces y tubérculos andinos: Una década de investigación para el desarrollo (19932003). N ${ }^{\circ}$. 8B. Lima, Peru: International Potato Center, Universidad Nacional Daniel Alcides Carrión, Erbacher Foundation, Swiss Agency for Development and Cooperation., 2005. 31p.

MARANGONI, A. L. Potencialidade de Aplicação de farinha de yacon (Polymnia sonchifolia) em produtos à base de cereais. 2007. 125f. Dissertação (Mestrado em Tecnologia de Alimentos) Universidade Estadual de Campinas. Faculdade de Engenharia de Alimentos, Campinas, SP.

MAYDATA.A.G.Café, antioxidantesyproteciónalasalud.Medisan, v.6, n.4, p.72-81, 2002. Disponível em: <http://www.bvs.sld.cu/ revistas/san/vol7_4_02/san11402.pdf>. Acesso em: 12. dez. 2013.

MOURA, N.A. et al. Protective effects of yacon (Smallanthus sonchifolius) intake on experimental colon carcinogenesis. Food and Chemical Toxicology, v.50, p.2902-2910, 2012. Disponível em: < http://dx.doi.org/10.1016/j.fct.2012.05.006>. Acesso em: 22 nov. 2013. doi: 10.1016/j.fct.2012.05.006

OLIVEIRA, M.A.; NISHIMOTO, E.K. Caracterização e quantificação dos carboidratos de reservas das raízes de yacon (Polymnia sonchifolia) mantidas sob condições ambientais e refrigeração. Revista Raízes e Amidos Tropicais, v.1, p.30-39, 2005. Disponível em: <http://energia.fca.unesp.br/index.php/rat/ article/view/1083>. Acesso em: 24 nov. 2013.

OLIVEIRA, M.A.; NISHIMOTO, E.K. Avaliação do desenvolvimento de plantas de yacon (Polymnia sonchifolia) e caracterização dos carboidratos de reservas em HPLC. Brazilian Journal of Food Technology, v.7, n.2, p.215-220, 2004. Disponível em: <http://bjft.ital.sp.gov.br/index.php>. Acesso em: 03 fev. 2014
OLIVEIRA, M.N. Tecnologia de produtos lácteos funcionais. São Paulo: Atheneu, 2009. 384p.

OLIVEIRA, G.O. et al. Improvement of biochemical parameters in type 1 diabetic rats after the roots aqueous extract of yacon (Smallanthus sonchifolius). Food and Chemical Toxicology, v.59, p.256-260, 2013. Disponível em: <http://dx.doi.org/10.1016/j. fct.2013.05.050>. Acesso em: 24 nov. 2013. doi: 10.1016/j. fct.2013.05.050.

PADILHA, V.M. et al. Perfil sensorial de bolos de chocolate formulados com farinha de yacon (Smallanthus sonchifolius) Ciência e Tecnologia de Alimentos, v.30, n.3, p.735-740, 2010. Disponível em: <http://www.scielo.br/pdf/cta/v30n3/v30n3a26. pdf $>$. Acesso em: 13 dez. 2013.

PEREIRA, D.I.; GIBSON, G.R. Effects of consumption of probiotics and prebiotics on sérum lipid levels in humans. Critica Reviews in Biochemistry and Molecular Biology, v.37, p.259281, 2002. Disponível em: <http://informahealthcare.com/doi/ pdf/10.1080/10409230290771519>. Acesso em: 12 dez. 2013. doi: 10.1080/10409230290771519.

QUINTEROS, E.T.T. Produção com tratamento enzimático e avaliação do suco de Yacon. 2000.163f. Tese (Doutorado em Tecnologia de Alimentos) - Universidade Estadual de Campinas, Faculdade de Engenharia de Alimentos, Campinas, SP.

RIBEIRO, J. de A. Estudos químico e bioquímico do yacon (Smallanthus sonchifolius) in natura e processado e influência de seu consumo sobre níveis glicêmicos e lipídeos fecais de ratos. 2008. 166f. Dissertação (Mestrado em Ciência dos Alimentos) Universidade Federal de Lavras, MG.

ROBERFROID, M.B. Inulin-Type fructans: functional food ingredients. Journal of Nutrition, v.137, p.24932502, 2007. Disponível em: <http://nutrition.highwire.org/ content/137/11/2493S.full.pdf+html>. Acesso em: 12 dez. 2013.

ROBINSON, H. Studies in the Heliantheae (Asteraceae). XII Re-establishment of the genus Smallanthus. Phytologia, v.39, n.1, p.47-53, 1997. Disponível em: <http://www.biodiversitylibrary. org/part/7608\#/summary>. Acesso em: 21 ago. 2014.

RODRIGUES, F.C. Avaliação da farinha de yacon (Smallanthus sonchifolius) na modulação das propriedades biomecânicas e na retenção de minerais nos ossos de ratos Wistar. 2011. 181f. Tese (Doutorado em Ciência e Tecnologia dos Alimentos) Universidade Federal de Viçosa, Viçosa, MG.

ROLIM, P.M. et al. Glycemic profile and prebiotic potential "in vitro" of bread with Yacon (Smallanthus sonchifolius) flour. Ciência e Tecnologia de Alimentos, v.31, n.2, p.467474, 2011. Disponível em: <http://dx.doi.org/10.1590/S010120612011000200029>. Acesso em: 12 dez. 2013 doi: 10.1590/ S0101-20612011000200029.

ROLIM, P.M. et al. Análise de componentes principais de pães de forma formulados com farinha de yacon (Smallanthus sonchifolius (Poepp.) H. Rob.). Revista Ceres, v.57, n.1, p.012-017, 2010. Disponível em: $<$ http://www.scielo.br/pdf/rceres/v57n1/a03v57n1. pdf $>$. Acesso em: 24 nov. 2013.

ROSA, C.S. et al. Elaboração de bolo com farinha de Yacon. Ciência Rural, v.39, n.6, p.1869-1872, 2009. Disponível em: $<$ http://dx.doi.org/10.1590/S0103-84782009005000131>. Acesso em: 12 nov. 2013. doi: 10.1590/S0103-84782009005000131. 
SAAD, S.M.I. Probióticos e prebióticos: o estado da arte. Revista Brasileira de Ciências Farmacêuticas, v.42, p.116, 2006. Disponível em: <http://dx.doi.org/10.1590/S151693322006000100002>. Acesso em: 12 nov. 2013. doi: 10.1590/ S1516-93322006000100002.

SANTANA, I.; CARDOSO, M.H. Raiz tuberosa de yacon (Smallanthus sonchifolius): potencialidade de cultivo, aspectos tecnológicos e nutricionais. Ciência Rural, v.38, n.3, p.898905, 2008. Disponível em: <http://www.scielo.br/scielo. php? script $=$ sci arttext\&pid $=$ S010384782008000300050\&lng $=\mathrm{e} \& \mathrm{nrm}=\mathrm{iso}>$. A . esso em: 06 out. 2013. doi: 10.1590/S010384782008000300050 .

SCHEID, M.M.A. Avaliação dos efeitos do consumo de yacon liofilizado em idosos. 2013. 122f. Tese (Doutorado em Ciência de Alimentos) - Universidade Estadual de Campinas, Faculdade de Engenharia de Alimentos, Campinas, SP.

SEMINARIO, J.; VALDERRAMA, M. El yacon: fundamentos para el aprovechamiento de un recurso promisorio. Lima, Peru: Centro Internacional de la Papa(CIP), Universidad Nacional de Cajamarca, Agencia Suiza para elDesarrolloy la Cooperación(COSUDE), 2003. 60p. Disponível em: <http://www.cipotato.org/market/PDFdocs/ Yacon_Fundamentos_password.pdf $>$. Acesso em: 02 dez. 2013.

SILVA, A.S.S. A raiz da yacon (Smallanthus sonchifollius Poepping \& Endlicher) como fonte de fibras alimentares, sua caracterização físico-química, uso na panificação e sua influência na glicemia pós-prandial. 2007. 158f. Tese (Doutorado em Ciência dos Alimentos) - Universidade Federal de Santa Catarina, Florianópolis, SC.

SILVA, E.B; CANDIDO, L.M.B. Processamento de bebida funcional à base de yacon (Polymnia sonchifolia Poepping \& Endlicher). 2004. 96f. Dissertação (Mestrado em Tecnologia de Alimentos) - Universidade Federal do Paraná (UFPR), Curitiba, PR.

SIMONOVSKA, B. et al. Investigation of phenolic acids in yacon (Smallanthus sonchifolius) leaves and tubers. Journal of Chromatography A, v.1016, n.1, p.89-98, 2003. Disponível em: $<$ http://dx.doi.org/10.1016/S0021-9673(03)01183-X>. Acesso em: 17 jan. 2014. doi: 10.1016/S0021-9673(03)01183-X.
TAKENAKA, M. Caffeic acid derivatives in the roots of yacon (Smallanthus sonchifolius). Journal of Agricultural and Food Chemistry, v.51, n.3, p.793-796, 2003. Disponível em: <http:// pubs.acs.org/doi/pdf/10.1021/jf020735i $>$. Acesso em: 12 nov: 2013. doi: pdf/10.1021/jf020735i.

TEIXEIRA, J.T. Elaboração de apresuntado formulado com farinha e extrato de yacon (Smallanthus sonchifollius). 2011. 114f. Dissertação (Mestrado em Ciência dos Alimentos) Universidade Federal de Lavras, Lavras, MG.

TOSTES, M.G.V. et al. Yacon effects in immune response and nutritional status of iron and zinc in preschool children. Nutrition, v.9142, p.666672, 2014. Disponível em: <http://www.sciencedirect.com/science/ article/pii/S0899900713004656>. Acesso em: 10 de jan. 2014.

VALENTOVÁ, K.; ULRICHOVÁ, J. Smallanthus sonchifolius and Lepidium meyenii - prospective Andean crops for the prevention of chronic diseases. Biomedical Papers, v.147, n.2, p.119-130, 2003. Disponível em: <http://mefanet.upol.cz/ BP/2003/2/119.pdf $>$. Acesso em: 12 nov. 2013.

VANINI, M. et al. A relação do tubérculo andino yacon com a saúde humana. Ciência Cuidado e Saúde, v.8, p.92-96, 2009. Disponível em: <http://periodicos.uem.br/ojs/index.php/ CiencCuidSaude/article/view/9723>. Acesso em: 20 jun. 2014. doi: 10.4025/cienccuidsaude.v8i0.9723.

VASCONCELOS, C.M. Caracterização físico-química e sensorial de iogurte "light" com farinha de yacon (Smallanthus sonchifolius). 2010. 56f. Dissertação (Mestrado em Ciência e Tecnologia de Alimentos) - Universidade Federal de Viçosa, Viçosa, MG.

VILHENA, S.M.C. et al. O cultivo do yacon no Brasil. Horticultura Brasileira, v.18, p.5-8, 2000. Disponível em: $<$ http://www.scielo. br/pdf/hb/v18n1/v18n1a_02.pdf >. Acesso em: 12 nov. 2013.

WANG, X.; GIBSON, G.R. Effects of the in vitro fermentation of oligofructose and inulin bybacteria growing in the human large intestine. Journal Applied Microbiology, v.74, n.4, p.373380, 1993. Disponível em: <http://onlinelibrary.wiley.com/ doi/10.1111/j.1365-2672.1993.tb02790.x/abstract>. Acesso em: 19 jun. 2014. doi: 10.1111/j.1365-2672.1993.tb02790.x. 